ORIGINAL ARTICLE

\title{
Clinical and cost effectiveness of paclitaxel, docetaxel, gemcitabine, and vinorelbine in non-small cell lung cancer: a systematic review
}

\author{
A Clegg, D A Scott, P Hewitson, M Sidhu, N Waugh
}

Thorax 2002;57:20-28

See end of article for authors' affiliations

........................

Correspondence to: $\operatorname{Dr}$ A Clegg Southampton Health Technology

Assessments Centre,

Wessex Institute for Health

Research and

Development, University of

Southampton, Southampton

SO16 7PX, UK

a.clegg@soton.ac.uk

Revised version received 8 August 2001

Accepted for publication

16 August 2001
Background: Lung cancer remains a devastating disease with few effective treatment options. Recent developments in chemotherapy have led to cautious optimism. This paper reviews the evidence on the clinical and cost effectiveness of four of the new generation drugs for patients with lung cancer.

Methods: A systematic review of randomised controlled trials (RCTs) identified from 11 electronic databases (including Medline, Cochrane library and Embase), reference lists and contact with experts and industry was performed to assess clinical effectiveness of paclitaxel, docetaxel, gemcitabine and vinorelbine. Clinical effectiveness was assessed using the outcomes of patient survival, quality of life, and adverse effects. Cost effectiveness was assessed by development of a costing model and presented as incremental cost per life year saved (LYS) compared with best supportive care (BSC).

Results: Of the 33 RCTs included, five were judged to be of good quality, 10 of adequate quality, and 18 of poor quality. Gemcitabine, paclitaxel, and vinorelbine as first line treatment and docetaxel as second line treatment appear to be more beneficial for non-small cell lung cancer than BSC and older chemotherapy agents, increasing patient survival by 2-4 months against BSC and some comparator regimes. These gains in survival do not appear to be at the expense of quality of life. Survival gains were delivered at reasonable levels of incremental cost effectiveness for vinorelbine, vinorelbine with cisplatin, gemcitabine, gemcitabine with cisplatin, and paclitaxel with cisplatin regimens compared with BSC. Conclusion: Although the clinical benefits of the new drugs appear relatively small, their benefit to patients with lung cancer appears to be worthwhile and cost effective.
D espite reductions over recent decades, lung cancer remains the leading cause of death from cancer and the third most common cause of all deaths in England and Wales with around 29000 deaths per annum. The outlook for patients following diagnosis is poor; $80 \%$ die within 1 year with only $5 \%$ surviving 5 years. ${ }^{1}$ Survival rates vary within England and Wales and across Europe. ${ }^{2}$

About $10 \%$ of patients with lung cancer are diagnosed early enough for cure by surgery, but most receive palliative care with radiotherapy and/or chemotherapy. Chemotherapy has often been considered toxic and ineffective, ${ }^{3}$ but recent developments have led to cautious optimism as a result of improvements in symptom relief, quality of life, and survival. ${ }^{4-9}$ It has been hoped that the new generation drugs such as paclitaxel, docetaxel, gemcitabine, and vinorelbine will provide sufficient benefit to dispel the nihilism surrounding lung cancer in the UK. ${ }^{10}$ Funding of chemotherapy varies among health authorities in England and Wales, partly due to uncertainties about their benefit but also because of concerns about the costs of the drugs and the possibility of realising any potential savings. ${ }^{11}$

In view of the continuing uncertainty over the clinical and cost effectiveness of the new chemotherapy agents and the "postcode prescribing" that has resulted, the National Institute for Clinical Excellence (NICE) was asked to provide national guidance for England and Wales. This paper summarises the results of a systematic review and economic evaluation commissioned to assist NICE in their deliberations on the clinical and cost effectiveness of paclitaxel, docetaxel, gemcitabine, and vinorelbine for patients with lung cancer. ${ }^{12}$

\section{METHODS}

Systematic review of clinical effectiveness

We searched for published studies in the English language using 11 electronic databases including Medline, Cochrane library, Embase, and Cancer Trials from their inception to December 2000 (search strategy reported elsewhere). ${ }^{12}$ Additional references including unpublished studies were sought by searching bibliographies of related publications and by contact with experts and industry. Studies reported only as abstracts or conference presentations were excluded.

Randomised controlled trials (RCTs) of paclitaxel, docetaxel, gemcitabine, and vinorelbine separately or in combination in the treatment of patients with lung cancer were included. Studies had to include either best supportive care (BSC), other new regimens, older regimens, or platinumbased combination regimens. The term BSC is used to describe care which includes relief of symptoms by, for example, analgesics, but which does not attempt to prolong life or to remove (even if only temporarily) the cause of the symptoms. BSC may vary in its inclusions. For example, radiotherapy may be part of palliative care by providing temporary relief of metastatic symptoms. Studies of chemotherapy as an addition to surgery or radiotherapy were excluded. Outcome measures included patient survival, quality of life, and adverse events. Tumour response was excluded from the review because of the poor correlation with symptom relief and patient survival. ${ }^{13}$

The quality of the RCTs was assessed using the Jadad scale. $^{14}$ This required cautious interpretation given the difficulties associated with blinding RCTs in chemotherapy, particularly when compared with BSC where the maximum score will be 3 rather than 5 . Inclusion criteria were applied, data were extracted, and quality was assessed by one reviewer and checked by a second reviewer, with any differences being resolved by consensus.

Clinical effectiveness was assessed using a narrative comparison of different outcomes including median survival, 
1 and 2 year survival, and differences in quality of life parameters. Meta-analysis was precluded by the diversity of interventions and comparators, differences in or insufficient details on outcomes used, patient characteristics, and drug dose and administration.

\section{Economic evaluation: the lung cancer costing model}

Given the preclusion of a meta-analysis and to make the analysis more robust, three modelling approaches were adopted: pairwise comparisons between the regimens (or BSC) from actual published trials; a cost minimisation analysis (CMA); and a cost effectiveness analysis (versus BSC) through synthesis of efficacy data by patient numbers. The pairwise and cost minimisation results are presented elsewhere. ${ }^{12}$ These were attempted for completeness but methodological concerns and the small size of the trials confounded the pairwise data while the usefulness of a CMA in policy decision making is limited.

\section{Sources of costs and resource utilisation}

Sixteen economic evaluations were found but none were UK based. A lack of readily available cost data hampered construction of a UK cost effectiveness model. Collection of costs was restricted to available published and unpublished data including detailed "bottom up" costing work done by the Scottish Health Purchasing Information Centre (SHPIC), the Scottish Health Service Cost's "blue book", and information from Southampton General Hospital. Unit costs are published elsewhere. ${ }^{12}$ Drug regimen costs were taken from the British National Formulary (BNF) using common trial dosages and based on a body surface area (BSA) of $1.7 \mathrm{~m}^{2}$. The cost of antiemetics and diuretics used in the trials was negligible and was excluded from the analysis. More modern drugs such as ondansetron are much more expensive but may also be more effective. Questions remain about the appropriate number of administrations per cycle and whether one cycle of one regimen is equivalent to one cycle of another. These points are also discussed elsewhere in detail. ${ }^{12}$ Best supportive care costs were based on data from case notes of 36 patients with stage IV non-small cell lung cancer (NSCLC) receiving terminal care, with adjustments for costs of inpatient care, outpatient care, home visits by primary care teams, and treatment costs relevant to BSC. ${ }^{12}$ This was the only known UK calculation of BSC. However, these data were cross checked against raw data from a larger series from the South-East Scotland Lung Study (SESLS). BSC estimates from SESLS were found to be similar to our previous figures. In the absence of available specific cost data on adverse events, a figure of $£ 500$ based on expert advice was added to account for such things as admission for drug induced neutropenia. This was applied irrespective of regimen, although it may vary between the four drugs with the cost for the taxanes perhaps being higher.

\section{Source of efficacy data}

Efficacy was analysed in terms of median survival since the response is not necessarily indicative of increased length of life. For the model, median survival by regimen was aggregated by patient numbers with larger trials thus carrying more weight. It is recognised that this method of pooling consists of indirect comparisons between trials and is therefore open to confounding. There may, for example, be differences in patient populations among trials. In addition, the comparator interventions vary markedly between trials and not all are in current usage. As a consequence, we chose median survival rather than incremental survival. However, the mixture of different patient types may also strengthen the conclusions and generalisability of the model. Although this approach is not the ideal way of directly comparing regimens, it does make the most of the data available, illustrates a range of possible cost effectiveness estimates across a range of assumptions, and can be interpreted with the aid of sensitivity analyses. Best and worst estimates were defined by the upper and lower bounds of individual trial data. Paclitaxel doses (and hence costs) varied markedly between the studies and so several regimens were modelled.

\section{Sensitivity analysis}

One way sensitivity analysis was carried out across a range of variables including number of cycles (advice from clinical colleagues was that in routine care a more realistic scenario would be to assume $60 \%$ of patients would have only $1-2$ cycles while $40 \%$ would continue towards the recommended number of cycles: three for gemcitabine, vinorelbine, and docetaxel regimens and four for paclitaxel); number of administrations per cycle of vinorelbine; best and worst cycles from trials; effect of discounts on BNF prices; and cost of newer antiemetic regimens. Mean survival estimates calculated from single studies by Berthelot et $a l^{15}$ and non-patient based utility estimates were also examined..$^{15}{ }^{16}$ The cost of BSC, particularly the number of inpatient days (21 versus 19 days), was varied to reflect slight differences between sources.

\section{RESULTS}

\section{Quantity and quality of clinical effectiveness studies}

Searching did not find any studies assessing the clinical effectiveness of the four drugs for treating small cell lung cancer. We included 33 RCTs to assess clinical effectiveness of the four drugs for treatment of NSCLC; three assessed docetaxel, ${ }^{14}{ }^{17-19}$ six gemcitabine, ${ }^{1320-27}$ six paclitaxel, ${ }^{28-33} 13$ vinorelbine, ${ }^{34-46}$ and five combined treatments. ${ }^{47-51}$ The characteristics of these studies are presented in table 1 .

Five RCTs were judged to be of good quality (Jadad score $\geqslant 2 / 3$ or $\geqslant 4 / 5$ ), 10 of adequate quality (Jadad score $3 / 5$ ), and 18 of poor quality (Jadad score $\leqslant 2 / 5$ ). Twenty RCTs lacked an adequate description of randomisation, ${ }^{17-23} 282932333542-44464749-51$ 32 had inadequate descriptions of blinding, ${ }^{13}$ 17-24 28-47 49-51 although for 18 this was difficult given the comparator (for example, BSC), 13 18-24313343-4749-51 and one did not describe withdrawals. ${ }^{24}$ Of the 33 RCTs, 15 stated that they were supported by or involved industry-two for docetaxel, ${ }^{17}{ }^{18}$ four for gemcitabine, ${ }^{13202124-26}$ three for paclitaxel, ${ }^{30} 3133$ and six for vinorelbine. $^{38-41} 4446$

\section{Clinical effectiveness of docetaxel}

Of the three RCTs (table 1), two compared docetaxel with BSC as either first ${ }^{18}$ or second line treatment, ${ }^{19}$ while the other compared docetaxel with vinorelbine or ifosfamide as second line treatment. ${ }^{17}$ Docetaxel appeared to increase median survival compared with BSC (table 2), although the benefit was only shown to be statistically significant when docetaxel $75 \mathrm{mg} / \mathrm{m}^{2}$ was used as second line treatment, improving median survival by nearly 3 months (docetaxel $75 \mathrm{mg} / \mathrm{m}^{2} 7.5$ months $v$ BSC 4.6 months, $\mathrm{p}=0.01) .{ }^{19}$ One year survival rates were significantly higher for patients given docetaxel 75 $\mathrm{mg} / \mathrm{m}^{2}(32 \%, 95 \%$ CI 23 to $40 ; \mathrm{p}<0.05)$ compared with vinorelbine or ifosfamide (19\%, 95\% CI 12 to 26$)$ as second line treatment. ${ }^{17}$ The effect of docetaxel on quality of life was assessed as first and second line therapy compared with BSC (table 3). ${ }^{18} 19$ As first line treatment, docetaxel had a limited effect on global health status and physical functioning but significantly improved emotional functioning $(\mathrm{p}<0.05)$, nausea/vomiting $(\mathrm{p}<0.05)$, pain $(\mathrm{p}<0.0001)$, and dyspnoea $(p<0.05) .{ }^{18}$ When used as second line treatment, docetaxel had a significant beneficial effect on pain $(p<0.01) .{ }^{19}$ Adverse effects varied between the different interventions. Haematological toxic events were more frequent among those receiving docetaxel than either BSC or vinorelbine or ifosfamide. In contrast, reporting of non-haematological toxic events differed little between docetaxel, BSC, and vinorelbine or ifosfamide. Higher toxic death rates were reported for patients 
Table 1 Characteristics of studies of clinical effectiveness

\begin{tabular}{|c|c|c|c|c|}
\hline Study details & Design & Intervention & Subjects & Conflicts of interest \\
\hline \multicolumn{5}{|l|}{ Docetaxel } \\
\hline $\begin{array}{l}\text { Shepherd et a }{ }^{19} \\
\text { Jadad quality score: } 2 / 3\end{array}$ & $\begin{array}{l}\text { Phase II, multicentre, randomised trial. } \\
\text { ITT }\end{array}$ & $\begin{array}{l}\text { Second line treatment: DOC } 100 \mathrm{mg} / \mathrm{m}^{2} \text { (49 patients), DOC } 75 \mathrm{mg} / \mathrm{m}^{2} \text { ( } 55 \text { patients), } \\
\text { and BSC (100 patients) }\end{array}$ & Stage IIIA/B or IV NSCLC & None stated \\
\hline $\begin{array}{l}\text { Roszkowski et all } \\
\text { Jadad quality score: } 2 / 3\end{array}$ & $\begin{array}{l}\text { Phase III, open-label, multicentre, } \\
\text { randomised trial. ITT }\end{array}$ & First line treatment: DOC $100 \mathrm{mg} / \mathrm{m}^{2}$ (137 patients) every 3 weeks, BSC (70 patients) & Stage IIIB or IV NSCLC & Supported by Rhone-Poulenc Rorer \\
\hline $\begin{array}{l}\text { Fossella et al } \\
\text { Jadad quality score: } 2 / 5 \\
\text { Gemcitabine }\end{array}$ & $\begin{array}{l}\text { Phase III, open label, multicentre, } \\
\text { randomised trial. ITT }\end{array}$ & $\begin{array}{l}\text { Second line treatment: DOC } 100 \mathrm{mg} / \mathrm{m}^{2} \text { (125 patients); DOC } 75 \mathrm{mg} / \mathrm{m}^{2}(125 \\
\text { patients); VNB or IFOS ( } 123 \text { patients) }\end{array}$ & NSCLC stage IIIB/IV & Supported by Rhone-Poulenc Rorer \\
\hline $\begin{array}{l}\text { Anderson et al }{ }^{13} \\
\text { Jadad quality score: } 3 / 3\end{array}$ & Multicentre, randomised trial. ITT & GEM $1000 \mathrm{mg} / \mathrm{m}^{2}$ with BSC (150 patients); BSC (150 patients) & $\begin{array}{l}\text { Symptomatic locally advanced or } \\
\text { metastatic NSCLC }\end{array}$ & Supported by Eli Lilly and Company \\
\hline $\begin{array}{l}\text { Bokkel Huinink et ap0 } 57 \\
\text { Jadad quality score: } 2 / 5\end{array}$ & $\begin{array}{l}\text { Phase II, multicentre, open-label, } \\
\text { randomised study. Not ITT }\end{array}$ & $\begin{array}{l}\text { GEM } 1000 \mathrm{mg} / \mathrm{m}^{2} \text { (72 patients); CDDP } 100 \mathrm{mg} / \mathrm{m}^{2} \text { with VP-16 } 100 \mathrm{mg} / \mathrm{m}^{2}(75 \\
\text { patients) }\end{array}$ & $\begin{array}{l}\text { Stage IIIA (inoperable), IIIB or IV } \\
\text { NSCLC }\end{array}$ & Supported by Eli Lilly and Company \\
\hline $\begin{array}{l}\text { Cardenal et a }{ }^{21} \\
\text { Jadad quality score: } 2 / 5\end{array}$ & $\begin{array}{l}\text { Phase III, multicentre, randomised } \\
\text { trial. ITT }\end{array}$ & GEM $1250 \mathrm{mg} / \mathrm{m}^{2}$ (69 patients); VP-16 $100 \mathrm{mg} / \mathrm{m}^{2}$ (66 patients) & Stage IIIB or IV NSCLC & Supported by Eli Lilly and Company \\
\hline $\begin{array}{l}\text { Crino et a }{ }^{22} \\
\text { Jadad quality score: } 2 / 5\end{array}$ & $\begin{array}{l}\text { Phase III, multicentre, randomised } \\
\text { trial. Partial ITT }\end{array}$ & $\begin{array}{l}\text { GEM } 1000 \mathrm{mg} / \mathrm{m}^{2} \text { with CDDP } 100 \mathrm{mg} / \mathrm{m}^{2} \text { (155 patients); MITO } 6 \mathrm{mg} / \mathrm{m}^{2} \text {, IFOS } \\
3000 \mathrm{mg} / \mathrm{m}^{2} \text {, with CDDP } 100 \mathrm{mg} / \mathrm{m}^{2} \text { (TriComb) (152 patients) }\end{array}$ & Stage IIIB or IV NSCLC & None stated \\
\hline $\begin{array}{l}\text { Perng et a }{ }^{33} \\
\text { Jadad quality score: } 3 / 5\end{array}$ & Phase II, randomised trial. ITT & $\begin{array}{l}\text { GEM } 1250 \mathrm{mg} / \mathrm{m}^{2} \text { (27 patients); CDDP } 80 \mathrm{mg} / \mathrm{m}^{2} \text { with VP- } 1680 \mathrm{mg} / \mathrm{m}^{2}(26 \\
\text { patients) }\end{array}$ & Stage III (A or B) or IV NSCLC & None stated \\
\hline $\begin{array}{l}\text { Sandler et a } P^{4} \\
\text { Jadad quality score: } 1 / 5 \\
\text { Paclitaxel }\end{array}$ & $\begin{array}{l}\text { Phase III, multicentre, randomised } \\
\text { trial. ITT }\end{array}$ & $\begin{array}{l}\text { GEM } 1000 \mathrm{mg} / \mathrm{m}^{2} \text { with CDDP } 100 \mathrm{mg} / \mathrm{m}^{2} \text { (260 patients); CDDP } 100 \mathrm{mg} / \mathrm{m}^{2} \text { (262 } \\
\text { patients) }\end{array}$ & Stage IIIA or IIIB or IV NSCLC & Supported by Eli Lilly \\
\hline $\begin{array}{l}\text { Bonomi et ap }{ }^{8} \\
\text { Jadad quality score: } 2 / 5\end{array}$ & $\begin{array}{l}\text { Phase III, multicentre, randomised } \\
\text { trial. Not ITT }\end{array}$ & $\begin{array}{l}\text { VP-16 } 100 \mathrm{mg} / \mathrm{m}^{2} \text { with CDDP } 75 \mathrm{mg} / \mathrm{m}^{2} \text { (193 patients); PAX } 250 \mathrm{mg} / \mathrm{m}^{2} \text { with CDDP } \\
75 \mathrm{mg} / \mathrm{m}^{2} \text { (191 patients); PAX } 135 \mathrm{mg} / \mathrm{m}^{2} \text { with CDDP } 75 \mathrm{mg} / \mathrm{m}^{2} \text { (190 patients) }\end{array}$ & Stage IIIB or IV NSCLC & None stated \\
\hline $\begin{array}{l}\text { Chang et a } R^{9} \\
\text { Jadad quality score: } 2 / 5\end{array}$ & Phase II, randomised study. Not ITT & $\begin{array}{l}\text { PAX } 250 \mathrm{mg} / \mathrm{m}^{2} \text { (25 patients); MER } 1000 \mathrm{mg} / \mathrm{m}^{2} \text { (35 patients); PIR } 150 \mathrm{mg} / \mathrm{m}^{2} \text { ( } 44 \\
\text { patients) }\end{array}$ & Stage IV NSCLC & None stated \\
\hline $\begin{array}{l}\text { Ranson et al } \\
\text { Jadad quality score: } 2 / 3\end{array}$ & $\begin{array}{l}\text { Phase III, multicentre, randomised } \\
\text { trial. ITT not stated }\end{array}$ & PAX $200 \mathrm{mg} / \mathrm{m}^{2}$ with BSC (79 patients); BSC (78 patients) & Stage IIIB or IV NSCLC & Supported by Bristol-Myers Squibb \\
\hline $\begin{array}{l}\text { Postmus et a } \text { P }^{2} \\
\text { Jadad quality score: } 2 / 5\end{array}$ & $\begin{array}{l}\text { Phase II, multicentre, randomised trial. } \\
\text { Interim analysis. ITT not stated }\end{array}$ & $\begin{array}{l}\text { CDDP } 80 \mathrm{mg} / \mathrm{m}^{2} \text { with VM-26 } 100 \mathrm{mg} / \mathrm{m}^{2} \text { (38 patients); PAX } 175 \mathrm{mg} / \mathrm{m}^{2} \text { and CDDP } \\
80 \mathrm{mg} / \mathrm{m}^{2} \text { (35 patients) }\end{array}$ & Stage IIIB or IV NSCLC & None stated \\
\hline $\begin{array}{l}\text { Gatzemier et a }{ }^{\beta 0} \\
\text { Jadad quality score: } 2 / 5\end{array}$ & $\begin{array}{l}\text { Phase III, multicentre randomised trial. } \\
\text { ITT }\end{array}$ & $\begin{array}{l}\text { PAX } 175 \mathrm{mg} / \mathrm{m}^{2} \text { with CDDP } 80 \mathrm{mg} / \mathrm{m}^{2} \text { (207 patients); CDDP } 100 \mathrm{mg} / \mathrm{m}^{2} \text { (207 } \\
\text { patients) }\end{array}$ & Stage IIIB or IV NSCLC & Supported by Bristol-Myers Squibb \\
\hline $\begin{array}{l}\text { Giaccone et a } a^{\beta 1} \\
\text { Jadad quality score: } 3 / 5 \\
\text { Vinorelbine }\end{array}$ & Phase III, randomised trial. ITT & $\begin{array}{l}\text { CDDP } 80 \mathrm{mg} / \mathrm{m}^{2} \text { with VM-26 } 100 \mathrm{mg} / \mathrm{m}^{2} \text { (166 patients); PAX } 175 \mathrm{mg} / \mathrm{m}^{2} \text { with CDDP } \\
80 \mathrm{mg} / \mathrm{m}^{2} \text { (166 patients) }\end{array}$ & $\begin{array}{l}\text { Locally advanced or metastatic } \\
\text { NSCLC }\end{array}$ & Supported by Bristol-Myers Squibb \\
\hline $\begin{array}{l}\text { Baldini et } a^{\beta 4} \\
\text { Jadad quality score: } 3 / 5\end{array}$ & $\begin{array}{l}\text { Phase II, multicentre, randomised } \\
\text { study. ITT }\end{array}$ & $\begin{array}{l}\text { CDDP } 80 \mathrm{mg} / \mathrm{m}^{2} \text { with VDS } 3 \mathrm{mg} / \mathrm{m}^{2} \text { and MITO } 6 \mathrm{mg} / \mathrm{m}^{2} \text { (49 patients); CDDP } 80 \\
\mathrm{mg} / \mathrm{m}^{2} \text { with IFOS } 3 \mathrm{mg} / \mathrm{m}^{2} \text { with VNB } 25 \mathrm{mg} / \mathrm{m}^{2} \text { (48 patients); CBDCA } 350 \mathrm{mg} / \mathrm{m}^{2} \\
\text { with VNB } 25 \mathrm{mg} / \mathrm{m}^{2} \text { (43 patients) }\end{array}$ & Stage IIIB or IV NSCLC & None stated \\
\hline $\begin{array}{l}\text { Colleoni et a }{ }^{\beta 5} \\
\text { Jadad quality score: } 1 / 5\end{array}$ & $\begin{array}{l}\text { Phase II, randomised trial. ITT not } \\
\text { stated }\end{array}$ & $\begin{array}{l}\text { CDDP } 100 \mathrm{mg} / \mathrm{m}^{2} \text { with MITO } 8 \mathrm{mg} / \mathrm{m}^{2} \text { and VNB } 25 \mathrm{mg} / \mathrm{m}^{2} \text { (26 patients); CBDCA } \\
400 \mathrm{mg} / \mathrm{m}^{2} \text { with VNB } 25 \mathrm{mg} / \mathrm{m}^{2} \text { (26 patients) }\end{array}$ & Stage IIIB and IV NSCLC & None stated \\
\hline $\begin{array}{l}\text { Colucci et a } \beta^{\beta} \\
\text { Jadad quality score: } 3 / 5\end{array}$ & $\begin{array}{l}\text { Phase III, multicentre, randomised } \\
\text { study. ITT }\end{array}$ & $\begin{array}{l}\text { Two step treatment arms - CDDP } 100 \mathrm{mg} / \mathrm{m}^{2} \text { with VNB } 25 \mathrm{mg} / \mathrm{m}^{2} \text {, followed by IFOS } \\
2.5 \mathrm{~g} / \mathrm{m}^{2} \text { and EPI } 100 \mathrm{mg} / \mathrm{m}^{2} \text { (53 patients). IFOS } 2.5 \mathrm{~g} / \mathrm{m}^{2} \text { and EPI } 100 \mathrm{mg} / \mathrm{m}^{2} \text {, } \\
\text { followed by CDDP } 100 \mathrm{mg} / \mathrm{m}^{2} \text { and VNB } 25 \mathrm{mg} / \mathrm{m}^{2} \text { (47 patients) }\end{array}$ & Stage IIIA/B and IV NSCLC & None stated \\
\hline $\begin{array}{l}\text { Comella et a }{ }^{\beta 7} \\
\text { Jadad quality score: } 3 / 5\end{array}$ & $\begin{array}{l}\text { Phase III, multicentre, randomised } \\
\text { trial. ITT }\end{array}$ & $\begin{array}{l}\text { CDDP } 40 \mathrm{mg} / \mathrm{m}^{2} \text { with VP-16 } 100 \mathrm{mg} / \mathrm{m}^{2} \text { (53 patients). CBDCA } 250 \mathrm{mg} / \mathrm{m}^{2} \text { with } \\
\text { CDDP } 30 \mathrm{mg} / \mathrm{m}^{2}, \text { VP-16 } 100 \mathrm{mg} / \mathrm{m}^{2} \text { and VNB } 30 \mathrm{mg} / \mathrm{m}^{2} \text { (52 patients) }\end{array}$ & Stage IIIB or IV NSCLC & None stated \\
\hline $\begin{array}{l}\text { Crawford et a } \beta^{\beta} \\
\text { Jadad quality score: } 3 / 5\end{array}$ & $\begin{array}{l}\text { Phase II, multicentre, randomised trial. } \\
\text { ITT }\end{array}$ & VNB $30 \mathrm{mg} / \mathrm{m}^{2}$ (143 patients). 5 -FU $425 \mathrm{mg} / \mathrm{m}^{2}$ with LV $20 \mathrm{mg} / \mathrm{m}^{2}$ (68 patients) & Stage IV NSCLC & Supported by Glaxo Wellcome \\
\hline $\begin{array}{l}\text { Depierre et a }{ }^{\beta 9} \\
\text { Jadad quality score: } 3 / 5\end{array}$ & $\begin{array}{l}\text { Phase III, multicentre, randomised } \\
\text { trial. ITT }\end{array}$ & VNB $30 \mathrm{mg} / \mathrm{m}^{2}$ (119 patients). VNB $30 \mathrm{mg} / \mathrm{m}^{2}$ with CDDP $80 \mathrm{mg} / \mathrm{m}^{2}$ (121 patients) & Stage IIIA/B or IV NSCLC & Supported by Pierre Fabre \\
\hline $\begin{array}{l}\text { Furuse et a a } \\
\text { Jadad quality score: } 3 / 5\end{array}$ & $\begin{array}{l}\text { Phase II, crossover, multicentre, } \\
\text { randomised trial. ITT not stated }\end{array}$ & $\begin{array}{l}\text { (VNB arm) VNB } 25 \mathrm{mg} / \mathrm{m}^{2} \text { (103 patients) with non-responders switching to VDS } \\
3 \mathrm{mg} / \mathrm{m}^{2}+C D D P 80 \mathrm{mg} / \mathrm{m}^{2} ; \text { (VDS arm) VDS } 3 \mathrm{mg} / \mathrm{m}^{2}(101 \text { patients) with } \\
\text { non-responders switching to VNB } 20 \mathrm{mg} / \mathrm{m}^{2}+\operatorname{CDDP} 80 \mathrm{mg} / \mathrm{m}^{2}\end{array}$ & Stage IIIB or IV NSCLC & $\begin{array}{l}\text { Supported by Kyowa Hakka } \\
\text { Company }\end{array}$ \\
\hline $\begin{array}{l}\text { Le Chevalier et a }{ }^{\mu 1} \\
\text { Jadad quality score: } 3 / 5\end{array}$ & $\begin{array}{l}\text { Phase II, international, multicentre, } \\
\text { randomised trial. ITT not stated }\end{array}$ & $\begin{array}{l}\text { VNB } 30 \mathrm{mg} / \mathrm{m}^{2} \text { with CDDP } 120 \mathrm{mg} / \mathrm{m}^{2} \text { (206 patients); VDS } 3 \mathrm{mg} / \mathrm{m}^{2} \text { with CDDP } \\
120 \mathrm{mg} / \mathrm{m}^{2} \text { (200 patients); VNB } 30 \mathrm{mg} / \mathrm{m}^{2} \text { (206 patients) }\end{array}$ & Stage III or IV NSCLC & Supported by Pierre Fabre \\
\hline $\begin{array}{l}\text { Lorusso et ali2 } \\
\text { Jadad quality score: } 2 / 5\end{array}$ & $\begin{array}{l}\text { Phase III, multicentre, randomised } \\
\text { trial. Not ITT }\end{array}$ & VNB $25 \mathrm{mg} / \mathrm{m}^{2}$ (35 patients); VNB $25 \mathrm{mg} / \mathrm{m}^{2}$ with CDDP $80 \mathrm{mg} / \mathrm{m}^{2}$ (34 patients) & Inoperable NSCLC & None stated \\
\hline
\end{tabular}




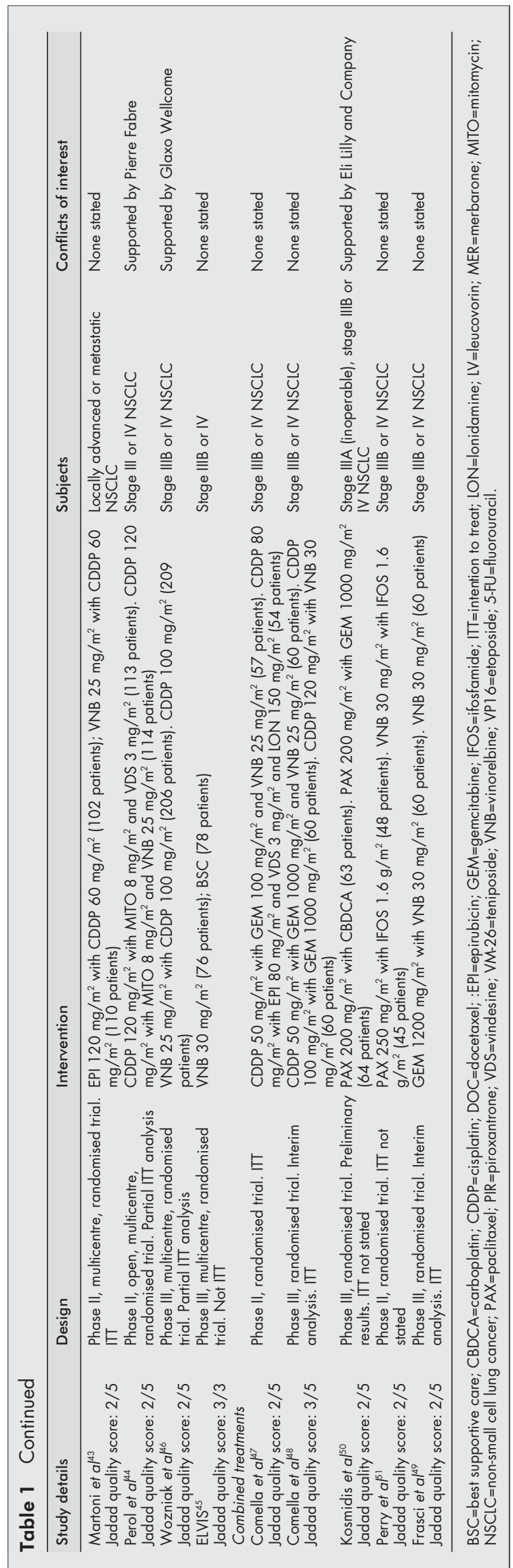

receiving $100 \mathrm{mg} / \mathrm{m}^{2}$ docetaxel, necessitating a reduction in dose to $75 \mathrm{mg} / \mathrm{m}^{2}{ }^{19}$

\section{Clinical effectiveness of gemcitabine}

Two of the six RCTs assessing gemcitabine used cisplatin and etoposide as comparator ${ }^{20}{ }^{23}$ while the other four RCTs compared gemcitabine and BSC with BSC alone, ${ }^{13}$ gemcitabine with etoposide, ${ }^{21}$ gemcitabine and cisplatin with cisplatin, ${ }^{24}$ and gemcitabine and cisplatin with mitomycin, ifosfamide and cisplatin (table 1 ). ${ }^{22}$ Gemcitabine ( 8.7 months, $95 \%$ CI 7.7 to 10.2 ) was shown to have a statistically significant benefit on the median survival of patients compared with etoposide (7.2 months, 95\% CI 6.1 to 9.8 ; $\mathrm{p}<0.05)^{21}$ and when combined with cisplatin (9.1 months, 95\% CI 8.3 to 10.6) compared with cisplatin alone (7.6 months, 95\% CI 6.5 to $8.2 ; \mathrm{p}<0.005$; table 2$).{ }^{24}$ Sustained improvements in measures of quality of life occurred significantly more frequently in patients receiving gemcitabine and BSC than in those treated with BSC alone (22\% v $9 \%$, $\mathrm{p}<0.005) .{ }^{13}$ Statistically significant changes to particular elements of the quality of life measures were evident (table 3 ). Patients receiving gemcitabine and cisplatin had significant improvements in chest pain $(\mathrm{p}<0.05)$, while those receiving mitomycin, ifosfamide and cisplatin $(\mathrm{p}<0.001)^{22}$ or etoposide

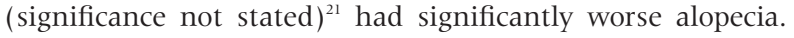
Adverse effects associated with gemcitabine differed little from the other drug comparators, but included grade 3 and 4 anaemia, neutropenia, thrombocytopenia, hair loss, nausea, infection, and diarrhoea.

\section{Clinical effectiveness of paclitaxel}

Six RCTs (table l) compared the clinical effectiveness of paclitaxel separately with merbarone and piroxantrone, ${ }^{29}$ as well as in several combinations including paclitaxel and cisplatin compared with etoposide with cisplatin, ${ }^{28}$ paclitaxel and BSC with $\mathrm{BSC}^{33}$ paclitaxel and cisplatin with teniposide and cisplatin, ${ }^{31}{ }^{32}$ paclitaxel and cisplatin against cisplatin. ${ }^{30}$ Paclitaxel and BSC ( 6.8 months, 95\% CI 5.7 to 10.2) were associated with statistically significant improvements in median survival compared with BSC (4.8 months, 95\% CI 3.7 to 6.8 ; $p<0.05$, table 2$).{ }^{33}$ One and two year survival was improved for patients receiving paclitaxel, ${ }^{28} 29133$ although only the comparison between paclitaxel and BSC (95\% CI 20 to 41) with BSC (95\% CI 18 to 39) was statistically significant. ${ }^{33}$ Of the four RCTs examining the effects of paclitaxel on quality of life (table 3 ), two found a significant beneficial effect on functional ability for patients receiving paclitaxel and BSC compared with BSC alone $(\mathrm{p}<0.05)^{33}$ and for paclitaxel and cisplatin compared with teniposide and cisplatin (fatigue $\mathrm{p}<0.01$, appetite loss $\mathrm{p}<0.001) .^{31}$ Adverse events, whether haematological or non-haematological, differed depending on the interventions compared. Three RCTs assessing paclitaxel with merbarone and piroxantrone, ${ }^{29}$ paclitaxel and cisplatin with cisplatin alone, $^{30}$ and paclitaxel and BSC with BSC only ${ }^{33}$ found that severe adverse effects were more frequent in patients receiving paclitaxel. In contrast, two of three RCTs comparing paclitaxel and cisplatin with teniposide and cisplatin showed severe adverse effects to be more evident in those on teniposide and cisplatin. ${ }^{31}{ }^{32}$ Adverse effects associated with paclitaxel included thrombocytopenia, leukopenia, anaemia, alopecia, and nausea/vomiting.

\section{Clinical effectiveness of vinorelbine}

Thirteen RCTs assessed 12 different comparisons of vinorelbine in combination with and in contrast to other interventions (table 1). Five RCTs compared different doses of vinorelbine and/or different combinations. ${ }^{3435} 394142$ Two RCTs used a form of crossover design, ${ }^{36}{ }^{40}$ although patients in one RCT only changed interventions when considered non-responders. ${ }^{40}$ Different combinations of vinorelbine were used in the RCTs 
Table 2 Summary of evidence of effect of docetaxel, gemcitabine, paclitaxel and vinorelbine on patient survival

\begin{tabular}{|c|c|}
\hline Study details & Patient survival \\
\hline \multicolumn{2}{|l|}{ Docetaxel } \\
\hline \multirow[t]{2}{*}{ Shepherd et al ${ }^{19}$} & $\begin{array}{l}\text { Median survival: } \mathrm{BSC}=4.6 \text { months }(95 \% \mathrm{Cl} 3.7 \text { to } 6.0) ; \mathrm{DOC} \text { (both doses })=7 \text { months }(95 \% \mathrm{Cl} 5.5 \text { to } 9.0)(\mathrm{p}=0.047) ; \mathrm{DOC}\left(100 \mathrm{mg} / \mathrm{m}^{2}\right)=5.9 \\
\text { months }(\mathrm{p}=0.78) ; \mathrm{DOC}\left(75 \mathrm{mg} / \mathrm{m}^{2}\right)=7.5 \text { months }(\mathrm{p}=0.01) \text {. }\end{array}$ \\
\hline & One year survival: BSC $=19 \% ;$ DOC (both doses) $=29 \% ;$ DOC $\left(100 \mathrm{mg} / \mathrm{m}^{2}\right)=19 \% ; \mathrm{DOC}\left(75 \mathrm{mg} / \mathrm{m}^{2}\right)=37 \% ; \mathrm{BSC}=12 \%$. \\
\hline \multirow[t]{3}{*}{ Roszkowski et al ${ }^{18}$} & Median survival: $\mathrm{DOC}$ arm $=6.0$ months $(95 \% \mathrm{Cl} 5.0$ to 8.0$)$; $\mathrm{BSC}$ arm $=5.7$ months (95\% $\mathrm{Cl} 4.4$ to 6.8$)$ \\
\hline & One year survival: $\mathrm{DOC}=25 \% ; \mathrm{BSC}=16 \%$ \\
\hline & Two year survival: $\mathrm{DOC}=12 \% ; \mathrm{BSC}=0 \%$ \\
\hline \multirow[t]{2}{*}{ Fossella et $a l^{17}$} & Median survival: DOC $100 \mathrm{mg} / \mathrm{m}^{2}=5.5$ months; $75 \mathrm{mg} / \mathrm{m}^{2}=5.7$ months; VNB or IFOS=5.6 months. \\
\hline & $\begin{array}{l}\text { One year survival: DOC } 100 \mathrm{mg} / \mathrm{m}^{2}=21 \%(95 \% \mathrm{Cl} 14 \text { to } 28 \%) \text {; DOC } 75 \mathrm{mg} / \mathrm{m}^{2}=32 \%(95 \% \mathrm{Cl} 23 \text { to } 40 \%) \text {; VNB or IFOS=19\% } 195 \% \text { Cl } 12 \text { to } \\
26 \%) \text {. }\end{array}$ \\
\hline \multicolumn{2}{|r|}{ 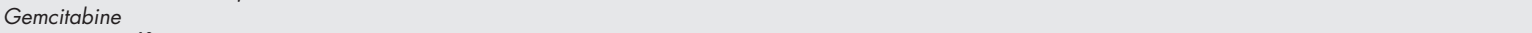 } \\
\hline \multirow[t]{3}{*}{ Anderson et al ${ }^{13}$} & Median survival: $\mathrm{GEM}+\mathrm{BSC}=5.7$ months $(95 \% \mathrm{Cl} 4.6$ to 7.6$) ; \mathrm{BSC}=5.9$ months $(95 \% \mathrm{Cl} 5.0$ to 7.9 ) \\
\hline & Estimated one year survival: GEM+BSC $=25 \%$; BSC $=22 \%$ \\
\hline & Estimated two year survival: $\mathrm{GEM}+\mathrm{BSC}=6 \%$; BSC $=7 \%$. \\
\hline \multirow{3}{*}{$\begin{array}{l}\text { Bokkel Huinink et } \\
\text { afo57 }\end{array}$} & Median survival: $\mathrm{GEM}=6.6$ months $(95 \% \mathrm{Cl} 4.9$ to 7.3$)$ \\
\hline & $\mathrm{CDDP}+\mathrm{VP}-16$ arm $=7.6$ months $(95 \% \mathrm{Cl} 5.4$ to 9.3$)$ \\
\hline & One year survival: $G E M=26 \% ; C D D P+V P-16$ arm $=24 \%(p=N S)$ \\
\hline \multirow[t]{2}{*}{ Cardenal et $a^{21}$} & Estimated median survival: $\mathrm{GEM}$ arm $=8.7$ months $(95 \% \mathrm{Cl} 7.7$ to 10.2$)$; VP-16 arm $=7.2$ months $(95 \% \mathrm{Cl} 6.1$ to 9.8$)(p=0.02)$. \\
\hline & One year survival probability: GEM=32\%; VP-16=26\% (p=NS). \\
\hline \multirow[t]{2}{*}{ Crino et $a^{22}$} & Overall median survival time: $\mathrm{GEM}+\mathrm{CDDP}=8.6$ months; TriComb=9.6 months ( $\mathrm{p}=\mathrm{NS})$. \\
\hline & One year survival: $\mathrm{GEM}+\mathrm{CDDP}=33 \%$; TriComb $=34 \%$ \\
\hline Perng et $a p^{3}$ & Median survival duration: $G E M=37$ weeks; $C D D P+V P-16=48$ weeks ( $p=N S$ ). \\
\hline & One year survival: not reported. \\
\hline Sandler et a ${ }^{24}$ & Estimated median survival: $\mathrm{GEM}+\mathrm{CDDP}=9.1$ months $(95 \% \mathrm{Cl} 8.3$ to 10.6$) ; \mathrm{CDDP}=7.6$ months $(95 \% \mathrm{Cl} 6.5$ to 8.2$)(\mathrm{p}<0.01)$ \\
\hline & Estimated one year survival: $\mathrm{GEM}+\mathrm{CDDP}=39 \% ; \mathrm{CDDP}=28 \%$ \\
\hline Paclitaxel & \\
\hline Bonomi et ap ${ }^{28}$ & Median survival: CDDP+VP-16=7.6 months; PAX $\left(250 \mathrm{mg} / \mathrm{m}^{2}\right)+C D D P=10$ months; $P A X\left(135 \mathrm{mg} / \mathrm{m}^{2}\right)+C D D P=9.5$ months \\
\hline & One year survival: $C D D P+V P-16=31.8 \% ; P A X\left(250 \mathrm{mg} / \mathrm{m}^{2}\right)+C D D P=40.3 \% ; P A X\left(135 \mathrm{mg} / \mathrm{m}^{2}\right)+C D D P=37.4 \%$ \\
\hline Chang et apq & Median survival: $P A X=24.1$ weeks; $M E R=19.9$ weeks; $P I R=29.3$ weeks $(p=N S)$. \\
\hline & One year survival: $P A X=m e a n(S D) 41.7(10) \% ; M E R=21.6(7) \% ; P I R=22.6(7) \%(p=N S)$ \\
\hline Ranson et $a^{\beta 3}$ & Median survival: $\mathrm{PAX}+\mathrm{BSC}=6.8$ months $(95 \% \mathrm{Cl} 5.7$ to 10.2$) ; \mathrm{BSC}=4.8$ months $(95 \% \mathrm{Cl} 3.7$ to 6.8$)$. \\
\hline & $\begin{array}{l}\text { One year survival: } \mathrm{PAX}+\mathrm{BSC}=95 \% \mathrm{Cl}: 20 ; 41 \%, \mathrm{BSC}=95 \% \mathrm{Cl}: 18 ; 39 \% . \mathrm{PAX}+\mathrm{BSC} \text { significantly associated with increased survival, hazard ratio } \\
0.68(95 \% \mathrm{Cl} 0.489 \text { to } 0.996 ; \mathrm{p}=0.048)\end{array}$ \\
\hline Postmus et $a^{\beta 2}$ & Survival: Not assessed. \\
\hline Gatzemier et $a^{\beta 0}$ & Survival: $\mathrm{PAX}+\mathrm{CDDP}=8.1$ months $(95 \% \mathrm{Cl} 7.3$ to 9.2$) ; \mathrm{CDDP}=8.6$ months $(95 \% \mathrm{Cl} 7.1$ to 10.3$)$. \\
\hline & Estimated one year survival: $P A X+C D D P=30 \% ; C D D P=36 \%$. \\
\hline Giaccone et $a^{\beta 1}$ & Median survival: $\mathrm{CDDP}+\mathrm{VM}-26=9.9$ months; $\mathrm{PAX}+\mathrm{CDDP}=9.7$ months $(\mathrm{p}=0.97)$ \\
\hline & One year survival: $\mathrm{CDDP}+\mathrm{VM}-26=41 \%(95 \% \mathrm{Cl} 33$ to $49 \%) ; \mathrm{PAX}+\mathrm{CDDP}=43 \%(95 \% \mathrm{Cl} 25$ to $51 \%)$ \\
\hline & Two year survival: $\mathrm{CDDP}+\mathrm{VM}-26=18 \%(95 \% \mathrm{Cl} 10$ to $26 \%), \mathrm{PAX}+\mathrm{CDDP}=19 \%(95 \% \mathrm{Cl} 12$ to $26 \%)$. \\
\hline Vinorelbine & \\
\hline Baldini et a ${ }^{\beta 4}$ & Median survival: $\mathrm{CDDP}+\mathrm{MITO}+\mathrm{VDS}=8.4$ months; $\mathrm{CDDP}+\mathrm{IFOS}+\mathrm{VNB}=8.8$ months; $\mathrm{CBDCA}+\mathrm{VNB}=7.9$ months. \\
\hline & One year survival: $C D D P+M I T O+V D S=18 \% ; C D D P+I F O S+V N B=15 \% ; C B D C A+V N B=16 \%$ \\
\hline Colleoni et a ${ }^{\beta 5}$ & Median survival: $\mathrm{CDDP}+\mathrm{MITO}+\mathrm{VNB}=9.9$ months (range 3-14); $\mathrm{CBDCA}+\mathrm{VNB}=8.8$ months (range 1-18). \\
\hline & One year survival: not assessed. \\
\hline Colucci et a $\beta^{\beta 6}$ & Median survival: $\mathrm{CDDP}+\mathrm{VNB}(\mathrm{IFOS}+\mathrm{EPI})=9$ months. IFOS+EPI (CDDP+VNB)=7 months $(\mathrm{p}=\mathrm{NS})$. \\
\hline & One year survival: not assessed. \\
\hline Comella et $a \beta^{\beta 7}$ & Median survival: $C D D P+V P-16=31$ weeks; $C B D C A+C D D P+V N B=27$ weeks $(p=N S)$ \\
\hline Crawford et $a^{\beta 8}$ & Median survival (estimated): $\mathrm{VNB}=30$ weeks; $5-\mathrm{FU}+\mathrm{LV}=22$ weeks $(\mathrm{p}=0.03)$ \\
\hline & One year survival: $\mathrm{VNB}=25 \% ; 5-\mathrm{FU}+\mathrm{LV}=16 \%(\mathrm{p}=0.06)$ \\
\hline Depierre et $a^{\beta 9}$ & Median survival: $\mathrm{VNB}=32$ weeks; $\mathrm{VNB}+\mathrm{CDDP}=33$ weeks $(\mathrm{p}=\mathrm{NS})$. \\
\hline & One year survival: not assessed. \\
\hline Furuse et $a l^{40}$ & Median survival: VNB arm=52.4 weeks; VDS arm=43.6 weeks ( $p=N S)$. \\
\hline & One year survival: not assessed. \\
\hline Le Chevalier et a ${ }^{41}$ & Median survival: $\mathrm{VNB}+\mathrm{CDDP}=40$ weeks; $\mathrm{VDS}+\mathrm{CDDP}=32$ weeks $(\mathrm{p}<0.09) ; \mathrm{VNB}=31$ weeks $(p<0.05)$ \\
\hline & One year survival: not assessed. \\
\hline Lorusso et $a l^{42}$ & Median survival: $\mathrm{VNB}=30$ weeks; $\mathrm{VNB}+\mathrm{CDDP}=38$ weeks $(\mathrm{p}=\mathrm{NS})$. \\
\hline & One year survival: not assessed. \\
\hline Martoni et $a^{43}$ & Median survival: $\mathrm{EPI}+\mathrm{CDDP}=10.5$ months $(95 \% \mathrm{Cl} 9.4$ to 11.5$)$; $\mathrm{VNB}+\mathrm{CDDP}=9.6$ months $(95 \% \mathrm{Cl} 8.4$ to 10.8$)$. \\
\hline & One year survival: $\mathrm{EPI}+\mathrm{CDDP}=42 \% ; \mathrm{VNB}+\mathrm{CDDP}=39 \%(\mathrm{p}=\mathrm{NS})$ \\
\hline & Two year survival: $\mathrm{EPI}+\mathrm{CDDP}=15 \% ; \mathrm{VNB}+\mathrm{CDDP}=8 \%(\mathrm{p}=\mathrm{NS})$ \\
\hline Perol et a ${ }^{44}$ & Median survival: $\mathrm{CDDP}+\mathrm{MITO}+\mathrm{VDS}=33.4$ weeks; $\mathrm{CDDP}+\mathrm{MITO}+\mathrm{VNB}=34.5$ weeks ( $\mathrm{p}=\mathrm{NS}$ ). \\
\hline & Overall two year survival: $C D D P+M I T O+V D S=15.6 \% ; C D D P+M I T O+V N B=9 \%$, ( $p=N S)$. \\
\hline Wozniak et $a^{46}$ & Median survival: $\mathrm{VNB}+\mathrm{CDDP}=8$ months, $\mathrm{CDDP}=6$ months $(\mathrm{p}<0.01)$ \\
\hline & One year survival: $\mathrm{VNB}+\mathrm{CDDP}=36 \% ; \mathrm{CDDP}=20 \%$. \\
\hline & Two year survival: $\mathrm{VNB}+\mathrm{CDDP}=12 \% ; \mathrm{CDDP}=6 \%$. \\
\hline Elderly Lung & Median survival: VNB 28 weeks; BSC 21 weeks. \\
\hline Cancer VNB Italian & 6 month survival: VNB $55 \%$; BSC $41 \%$. \\
\hline Study Group & One year survival: VNB 32\%; BSC 14\%. \\
\hline Combined treatmen & \\
\hline Comella et a ${ }^{47}$ & Median survival: $C D D P+G E M+V N B=50$ weeks $(95 \% \mathrm{Cl} 41$ to 58$) ; C D D P+E P I+V D S+L O N=33$ weeks $(95 \% \mathrm{Cl} 24$ to 41$)$ \\
\hline & One year survival: $C D D P+G E M+V N B=48 \% ; C D D P+E P I+V D S+L O N=29 \%$ \\
\hline & Two year survival: $\mathrm{CDDP}+\mathrm{GEM}+\mathrm{VNB}=19 \%, \mathrm{CDDP}+\mathrm{EPI}+\mathrm{VDS}+\mathrm{LON}=0 \%$ \\
\hline Comella et $a^{48}$ & Median survival: $C D D P+G E M+V N B=51$ weeks; $C D D P+G E M=42$ weeks; $C D D P+V N B=35$ weeks \\
\hline & One year survival: $C D D P+G E M+V N B=45 \% ; C D D P+G E M=40 \% ; C D D P+V N B=34 \%$ \\
\hline Kosmidis et $a^{50}$ & Median survival: not assessed. \\
\hline & One year survival: not assessed. \\
\hline Perry et $a^{51}$ & Median survival: $\mathrm{PAX}+\mathrm{IFOS}=8.5$ months; $\mathrm{VNB}+\mathrm{IFOS}=7.4$ months (95\% $\mathrm{Cl} 5.3$ to 13.3 ). \\
\hline & One year survival (estimated): PAX+IFOS=35\% (95\% Cl: $24 ; 52 \%) ; \mathrm{VNB}+\mathrm{IFOS}=38 \%(95 \% \mathrm{Cl} 26$ to $55 \%)$. \\
\hline Frasci et a ${ }^{49}$ & Median survival: $\mathrm{GEM}+\mathrm{VNB}=29$ wks; $\mathrm{VNB}=18$ weeks. \\
\hline & Six month survival (estimated): GEM+VNB $=56 \%$; VNB $=32 \%$. \\
\hline & One year survival (estimated): GEM+VNB $=30 \%$; VNB $=13 \%$. \\
\hline
\end{tabular}

$B S C=$ best supportive care; $C B D C A=$ carboplatin; $C D D P=$ cisplatin; $D O C=$ docetaxel; :EPI=epirubicin; $G E M=$ gemcitabine; IFOS=ifosfamide; ITT=intention to treat; LON=lonidamine; $L V=$ leucovorin; $M E R=$ merbarone; $M I T O=$ mitomycin; NSCLC=non-small cell lung cancer; $P A X=$ paclitaxel; $P I R=$ piroxantrone; VDS=vindesine; VM-26=teniposide; VNB=vinorelbine; VP-16=etoposide; 5 -FU=fluorouracil. 
Table 3 Summary of evidence of effect of docetaxel, gemcitabine, paclitaxel and vinorelbine on quality of life

\begin{tabular}{|c|c|}
\hline Study details & Quality of life \\
\hline \multicolumn{2}{|l|}{ Docetaxel } \\
\hline Shepherd et al19 & $\begin{array}{l}\text { QoL parameters favoured DOC patients, significant differences for pain }(p=0.006) \text {, fatigue }(p=0.06) \text { and tumour related } \\
\text { medications used }(p=0.02) \text {. }\end{array}$ \\
\hline Roszkowski et al ${ }^{18}$ & $\begin{array}{l}\text { DOC had significantly favourable effects on emotional functioning ( } p<0.05) \text {, nausea/vomiting }(p=0.04) \text {, pain }(p<0.0001) \text { and } \\
\text { dyspnea ( } p=0.02) \text {. No difference between global health status and physical functioning scores }(p=N S) \text {. }\end{array}$ \\
\hline \multicolumn{2}{|r|}{ 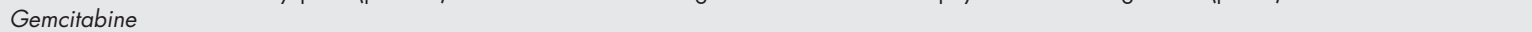 } \\
\hline Anderson et $a l^{13}$ & $\begin{array}{l}\text { On SS14 symptom scale GEM+BSC patients improved }(-10 \%) \text { from baseline to } 2 \text { months compared with deterioration in BSC } \\
\text { patients }(+1 \%)(p=0.113) \text {. Sustained }(\geqslant 4 \text { weeks) improvement }(\geqslant 25 \%) \text { in SS14 score was significantly higher for patients on } \\
\text { GEM+BSC }(22 \%) \text { compared with BSC }(9 \%)(p<0.005)\end{array}$ \\
\hline Bokkel Huinink et apo 57 & $\begin{array}{l}\text { No significant difference in change from baseline on global, physical, role, cognitive, emotional and social aspects of Qol } \\
(p>0.05) \text {. }\end{array}$ \\
\hline Cardenal et a ${ }^{p 1}$ & $\begin{array}{l}\text { No clinically significant differences in change from baseline within treatment arm or between treatment arms in functional } \\
\text { domains or global QoL. Statistically significant difference between treatment arms in change from baseline for alopecia, worse } \\
\text { for the VP-16 arm. Pain, insomnia, cough, hemoptysis, chest pain and shoulder pain by GEM and VP-16. }\end{array}$ \\
\hline Crino et $a^{p^{2}}$ & $\begin{array}{l}\text { Global QoL did not change significantly in either arm. Comparisons of change from baseline showed a worsening of alopecia } \\
\text { in the TriComb arm and a greater improvement in chest pain in the GEM+CDDP arm }(p<0.05) \text {. }\end{array}$ \\
\hline Sandler et a ${ }^{24}$ & No significant differences in QoL between treatment arms in change from baseline. \\
\hline \multicolumn{2}{|l|}{ Paclitaxel } \\
\hline Bonomi et a $\mathrm{p}^{8}$ & No significant difference between treatment arms in change from baseline. \\
\hline Ranson et $a^{\beta 3}$ & No statistically significant difference between arms in change from baseline. \\
\hline Gatzemier et a ${ }^{\beta 0}$ & $\begin{array}{l}\text { On symptom scales CDDP patients had significant worsening of nausea and vomiting }(p<0.0003) \text {, appetite loss }(p<0.02) \text { and } \\
\text { constipation }(p<0.032) \text {, while PAX+CDDP patients had significant worsening of hair loss and peripheral neuropathy } \\
(p<0.0001) \text {. }\end{array}$ \\
\hline Giaccone et $a^{\beta 1}$ & $\begin{array}{l}\text { Patients on PAX+CDDP had significant beneficial effects on functional scales and some symptom scales at } 6 \text { weeks (fatigue } \\
(p=0.006) \text { and appetite loss }(p<0.001)) \text {, which disappeared at } 12 \text { weeks. }\end{array}$ \\
\hline \multicolumn{2}{|r|}{ 童 } \\
\hline Crawford et a $\beta^{\beta 8}$ & No significant difference between treatment arms in change from baseline (no data presented). \\
\hline Martoni et $a^{43}$ & No significant difference in change from baseline between treatment arms. \\
\hline ELVIS ${ }^{45}$ & $\begin{array}{l}\text { On EORTC functional and symptom scales and on LC-13, VNB had significant improvement in cognitive function }(p=0.02) \text {, pain } \\
(p=0.02) \text {, dyspnea }(p=0.05) \text {, and pain medication }(p=0.01) \text {, but significantly worse on constipation }(p=0.002) \text {, nausea and } \\
\text { vomiting }(p=0.07) \text { peripheral neuropathy }(p=0.04) \text { and hair loss }(p=0.0001) \text {. }\end{array}$ \\
\hline \multicolumn{2}{|l|}{ Combined treatments } \\
\hline Comella et a ${ }^{47}$ & Improved QoL score CDDP+GEM+VNB=59\%,CDDP+EPI+VDS+ LON=39\% (p not stated) \\
\hline Frasci et a ${ }^{49}$ & $\begin{array}{l}\text { Almost } 60 \% \text { of GEM+VNB patients did not show impairment of QoL during treatment, compared to approximately } 40 \% \text { in the } \\
\text { VNB arm. Insufficient reporting of QoL measures ( } p \text { not stated). }\end{array}$ \\
\hline
\end{tabular}

including vinorelbine alone; vinorelbine and cisplatin; vinorelbine and carboplatin; vinorelbine, carboplatin and cisplatin; vinorelbine, mitomycin and cisplatin; vinorelbine, cisplatin and ifosfamide; vinorelbine, cisplatin, ifosfamide and epirubicin; and vinorelbine, cisplatin, carboplatin and etoposide. One RCT concentrated on elderly patients aged over 70 years. ${ }^{45}$ Of the 11 RCTs showing improvement in median survival for patients receiving vinorelbine in differing combinations, ${ }^{34-36}$ 38-42 44-46 the comparisons of vinorelbine with fluorouracil and leucovorin (30 weeks $v 22$ weeks, p<0.05) and vinorelbine and cisplatin with cisplatin (8 months $v 6$ months, $\mathrm{p}<0.005)$ showed statistically significant increases in survival (table 2). ${ }^{38} 46$ Patient survival to 1 and 2 years was assessed in six RCTs with none showing a significant difference between the combinations of interventions. ${ }^{34} 38$ 43-46 The effect of vinorelbine on quality of life was assessed in three RCTs (table 3), ${ }^{38435}$ although only the comparison between vinorelbine and BSC showed any statistically significant difference. ${ }^{45}$ Patients receiving vinorelbine experienced significant improvements in cognitive function $(p<0.05)$, dyspnoea $(p=0.05)$, and pain medication $(p=0.01)$, but significant worsening in constipation $(p<0.005)$, peripheral neuropathy $(\mathrm{p}<0.05)$, and hair loss $(\mathrm{p}<0.001)$. Adverse events, including constipation, heart toxicity, leukopenia, neutropenia, vomiting and alopecia, varied with the different combinations compared. Only two RCTs found any significant variation. ${ }^{43}{ }^{44}$ When compared with vinorelbine and cisplatin, patients receiving epirubicin and cisplatin suffered significantly more leukopenia $(\mathrm{p}=0.01)$, thrombocytopenia $(\mathrm{p}<0.05)$, and alopecia $(\mathrm{p}=0.001) .{ }^{43}$ Patients receiving vinorelbine, mitomycin, and cisplatin suffered significantly more anaemia $(p<0.01)$, neutropenia $(p<0.01)$, sepsis $(p<0.05)$, and local reaction $(\mathrm{p}<0.05)$ than those receiving vindesine, mitomycin, and cisplatin. ${ }^{44}$ In addition, five patients stopped treatment because of severe toxic events in the comparison of vinorelbine with BSC. ${ }^{45}$

\section{Clinical effectiveness of other combined treatments}

Of the five RCTs assessing the clinical effectiveness of combined treatments, two compared cisplatin, gemcitabine and vinorelbine with either cisplatin epirubicin, vindesine and lonidamine $e^{47}$ or cisplatin and gemcitabine and cisplatin and vinorelbine (table 1$){ }^{48}$ Other RCTs compared paclitaxel and ifosfamide with vinorelbine and ifosfamide, ${ }^{51}$ gemcitabine and vinorelbine with vinorelbine, ${ }^{49}$ and paclitaxel and carboplatin with paclitaxel and gemcitabine..$^{50}$ Only the combination of cisplatin, gemcitabine and vinorelbine ( 50 weeks, $95 \%$ CI 41 to 58) compared with cisplatin, epirubicin, vindesine and lonidamine ( 33 weeks, 95\% CI 24 to 41 ) was associated with a statistically significant increase in median survival (table 2). ${ }^{47}$ Assessment of the effects on quality of life was limited, with none of the combined treatments affecting quality of life (table 3)..$^{47-49}{ }^{51}$ Adverse effects varied with the components of the combined treatments, although no significant differences were evident. ${ }^{47-51}$

\section{The lung cancer costing model}

The results are presented in terms of incremental cost per life year saved (tables 4 and 5) using the synthesis of trial data to give a broad picture of likely relative cost effectiveness compared with BSC. Only the single new agents and their combination with cisplatin have been considered. BSC is the comparator as this remains standard treatment for most patients in the UK. Caution should be used in any comparison of regimens because of the way the data were combined (described above) and the lack of direct comparisons. 
Table 4 Cost effectiveness results $†$

\begin{tabular}{|c|c|c|c|c|c|c|c|c|c|c|c|}
\hline & BSC & GEM & $\begin{array}{l}\text { GEM+ } \\
\text { CDDP }\end{array}$ & VNB & $\begin{array}{l}\text { VNB+ } \\
\text { CDDP }\end{array}$ & PAX & $\begin{array}{l}\text { PAX } \\
(135)^{*} \\
+ \text { CDDP }\end{array}$ & $\begin{array}{l}\text { PAX } \\
(175)^{*} \\
+ \text { CDDP }\end{array}$ & $\begin{array}{l}\text { PAX } \\
(250)^{*} \\
+ \text { CDDP }\end{array}$ & DOC & $\mathrm{DOC}(2 \mathrm{~L})$ \\
\hline Median no of cycles & & 3 & 4 & 3 & 3 & 5 & 5 & 5 & 4 & 3 & 3 \\
\hline No. of administrations (GEM, VNB, etc) & & 9 & 12 & 12 & 12 & 5 & 5 & 5 & 4 & 3 & 3 \\
\hline No. of CDDP administrations & & 0 & 4 & 0 & 3 & 0 & 5 & 5 & 4 & 0 & 0 \\
\hline Drug cost (GEM,VNB, etc) ( $($ ) & & 2637 & 3516 & 2140 & 2140 & 6858 & 4364 & 5610 & 6483 & 3975 & 3300 \\
\hline Drug cost (CDDP) (£) & & & 243 & & 219 & & 243 & 243 & 204 & & \\
\hline Administration/side effects/chem counselling ( $($ ) & & 1495 & 2562 & 1823 & 2377 & 1435 & 1696 & 1696 & 1460 & 1065 & 1065 \\
\hline Average cost per patient $(£)$ & 3342 & 4132 & 6321 & 3963 & 4736 & 8293 & 6304 & 7550 & 8147 & 5040 & 4365 \\
\hline Incremental cost $(v$ BSC) $(£)$ & & 789 & 2979 & 620 & 1394 & 4951 & 2962 & 4208 & 4804 & 1698 & 1023 \\
\hline Media & 5.24 & 6.90 & 8.80 & 7.06 & 8.4 & 6.5 & 9.40 & 8.81 & 10.00 & 6.00 & 5.94 \\
\hline Life years s & 0.44 & 0.58 & 0.73 & 0.59 & 0.70 & 0.54 & 0.78 & 0.73 & 0.83 & 0.50 & 0.49 \\
\hline Average cost per LYS (£) & 7658 & 7184 & 8623 & 6738 & 6726 & 15283 & 8048 & 10281 & 9776 & 10081 & 8824 \\
\hline Incremental median surviv & & 1.66 & 3.56 & 1.82 & 3.2 & 1.27 & 4.1 & 3.58 & 4.76 & 0.76 & 0.70 \\
\hline Incremental LYS ( $v$ BSC) & & 0.14 & 0.30 & 0.15 & 0.27 & 0.11 & 0.35 & 0.30 & 0.40 & 0.06 & 0.06 \\
\hline Incremental cost per LYS (v BSC) (£) & & 5690 & 10041 & 4091 & 5206 & 46610 & 8537 & 14124 & 12104 & 26707 & 17546 \\
\hline
\end{tabular}

$\mathrm{BSC}=$ best supportive care; GEM=gemcitabine; VNB=vinorelbine; $\mathrm{PAX}=$ paclitaxel; $\mathrm{DOC}=$ docetaxel; $C D D P=$ cisplatin; $\mathrm{LYS}$ =life years saved; $2 \mathrm{~L}=$ second line treatment.

*Dose in $\mathrm{mg} / \mathrm{m}^{2}$. †All costs obtained in or converted to $1999 / 2000$ prices. ${ }^{58}$

Table 5 Selected one way sensitivity analysis: incremental cost per LYS $(£) \vee$ BSC

\begin{tabular}{|c|c|c|c|c|c|c|c|c|c|c|}
\hline & GEM & $\begin{array}{l}\text { GEM+ } \\
\text { CDDP }\end{array}$ & VNB & $\begin{array}{l}\text { VNB+ } \\
\text { CDDP }\end{array}$ & PAX & $\begin{array}{l}\text { PAX } \\
(135) \dagger \\
+ \text { CDDP }\end{array}$ & $\begin{array}{l}\text { PAX } \\
(175) \dagger \\
+ \text { CDDP }\end{array}$ & $\begin{array}{l}\text { PAX } \\
(250) \dagger \\
+ \text { CDDP }\end{array}$ & $\mathrm{DOC}$ & $\mathrm{DOC}(2 \mathrm{~L})$ \\
\hline Baseline & 5690 & 10041 & 4091 & 5206 & 46610 & 8537 & 14124 & 12104 & 26707 & 17546 \\
\hline 3 cycles: VNB,GEM,DOC; 4 PAX & 5690 & 5145 & 4091 & 5206 & 31957 & 5198 & 9398 & 12104 & 26707 & 17546 \\
\hline $60 \%$ prob. half course, $40 \%$ full course & D & 2478 & D & 2808 & 16461 & 2179 & 4879 & 7204 & 7952 & 569 \\
\hline VNB cycle: 21 days $/ 3$ administrations & NA & NA & D & 1982 & NA & NA & NA & NA & NA & NA \\
\hline VNB cycle: 21 days $/ 2$ administrations & NA & NA & D & D & NA & NA & NA & NA & NA & NA \\
\hline $25 \%$ discount on $B N F$ & 939 & 7079 & 564 & 3208 & 30469 & 5392 & 9416 & 8021 & 11078 & 3398 \\
\hline $50 \%$ discount on BNF & D & 4116 & D & 1209 & 14327 & 2248 & 4708 & 3937 & D & D \\
\hline Anti-emetics $£ 150$ & 6771 & 10547 & 5080 & 5767 & 48023 & 8970 & 14627 & 12482 & 29066 & 20119 \\
\hline Bethelot's mean survival ${ }^{15}$ & 1925 & NA & 2216 & 3167 & NA & 5196 & NA & 8429 & NA & NA \\
\hline Berthelot's QoL utilities ${ }^{15}$ & 5538 & NA & 5105 & 7290 & NA & 11296 & NA & 16358 & NA & NA \\
\hline Best survival & 2903 & 9253 & 3442 & 3833 & 38008 & NA & 11314 & NA & 7375 & 5425 \\
\hline Worst survival & 20458 & 12484 & 5895 & 11430 & 163647 & NA & 17636 & NA & $x$ & 46680 \\
\hline SESLS BSC cost (£3572) & 4034 & 9267 & 2576 & 4348 & 44447 & 7875 & 13352 & 11525 & 23093 & 13605 \\
\hline Lower BSC estimate ( $£ 2200$ ) & 13923 & 13892 & 11622 & 9473 & 57364 & 11830 & 17957 & 14982 & 44670 & 37134 \\
\hline Best cycles & D & NA & NA & NA & NA & NA & NA & NA & NA & NA \\
\hline Worst cycles & 23089 & 14938 & 11677 & 10467 & NA & NA & 18849 & NA & & NA \\
\hline OP administration & NA & 8255 & NA & 3722 & 43058 & NA & NA & NA & 23146 & 13664 \\
\hline Reduced dose (if $<20 \%$ of vial used) & NA & NA & 1618 & 3805 & NA & NA & NA & NA & NA & NA \\
\hline
\end{tabular}

$B S C=$ best supportive care; $G E M=$ gemcitabine; $V N B=$ vinorelbine; $P A X=$ paclitaxel; $D O C=$ docetaxel; $C D D P=c i s p l a t i n ; L Y=l i f e$ years saved; $2 \mathrm{~L}=$ second line treatment; SESLS=South-east Scotland Lung Study; $D=$ dominant strategy $v$ BSC. It is not appropriate to show a figure when a strategy is dominant; $X=$ dominated, higher cost and lower survival than BSC.

Data in italics were provided by industry.

*Cisplatin components not discounted; †dose in $\mathrm{mg} / \mathrm{m}^{2}$.

The regimens with the least incremental cost effectiveness over BSC under the baseline scenario are vinorelbine, vinorelbine+cisplatin, and gemcitabine. These regimens retain their cost effectiveness under a range of assumptions and may even be dominant under certain circumstances. The gemcitabine and vinorelbine regimens deliver similar levels of cost effectiveness if the same number of cycles and cycle length are applied. However, the results also show the reasonable cost effectiveness of gemcitabine+cisplatin and the paclitaxel+cisplatin regimens compared with BSC throughout a range of scenarios and assumptions. The (unlicensed) paclitaxel and docetaxel single agents remain relatively expensive compared with BSC. Docetaxel appears to be relatively expensive as second line treatment in the baseline scenario because of its small survival gain over BSC, but would be prescribed for only small numbers of patients.

Costs in routine care would probably be much lower than those based on data from trials. In the trials patients would be given chemotherapy as per the protocol if they could tolerate it, whereas in routine care physicians and patients would review continuation on a course by course basis, with chemotherapy being stopped in those whose tumours did not respond. This would make chemotherapy much more cost effective (see line 3, table 5).

\section{DISCUSSION}

Evidence of clinical effectiveness appeared to be of reasonable quality given the difficulties associated with blinding many of the treatments. Gemcitabine, paclitaxel, and vinorelbine as first line treatment and docetaxel as second line treatment appear to be beneficial to patient survival and to quality of life, particularly when used as combined treatments. Although improvements in median survival tend to be relatively small, ranging from 2 to 4 months, these appear worthwhile given that survival for untreated patients tends to be limited to 
about 5 months. Importantly, these gains in survival are not at the expense of quality of life which appears to have improved compared with BSC or the older chemotherapy agents.

Vinorelbine, vinorelbine + cisplatin, and gemcitabine appear to have the least incremental cost relative to BSC, taking into account both survival gains and quality of life. Were higher levels of funding available, the increased survival offered by the gemcitabine + cisplatin and paclitaxel+cisplatin regimens could be favoured. However, given the opportunity of informed choice, not all patients would wish to undergo treatment other than for palliative care. For example, a survey of 81 patients by Silvestri et al $l^{52}$ reported that patients would not want chemotherapy unless median survival improved by 4.5 months for mild toxicity and 9.0 months for severe toxicity. However, one of our expert reviewers reported a lack of understanding by patients on the effects and side effects of chemotherapy, noting a general belief that the side effects of such treatments outweigh any benefits. It was not possible to present results by disease stage given the lack of subgroup analysis in the reporting of survival data, although the majority of patients were stage IV.

Consistent methods for undertaking systematic reviews were applied throughout the review, ${ }^{53}$ with support from an expert advisory group including clinicians, patient representatives, and academics. Possible limitations were lack of follow up with authors to clarify study details, use of the Jadad scale for assessing methodological quality when it may more accurately reflect how well a study was reported, ${ }^{54}$ and lack of a validated method for assessing the methodological quality of quality of life studies.

Possible inadequacies in individual studies may undermine the evidence of effectiveness. Although nearly half of the studies examined quality of life as a primary or secondary outcome, very few evaluated it adequately, limiting accurate assessment of clinical and cost effectiveness. Studies provided limited information on patient characteristics, affecting any assessment among different patient subgroups or the generalisability of findings to patients referred in practice. Some studies failed to report results using intention to treat analysis which, when coupled with the high attrition of patients, creates the opportunity for bias. Several studies were either sponsored or undertaken by the manufacturers of the drugs which may bring into question their independence and lead to fears of bias. ${ }^{55}$

The new drugs represent a worthwhile but still very modest advance, with no cure and a gain in survival of only a few months. However, when valuing short durations of life, it has been argued that the concept of diminishing marginal utility weighting should reflect the fact that patients value a short extension to a short life expectancy more than a short extension to a longer life expectancy. ${ }^{56}$ Further research is needed including good quality RCTs of different combinations of treatments among different subgroups of patients; use of these regimens alongside radiotherapy for suitable patients; adequate assessment of quality of life; development of methods for assessing the methodological quality of quality of life studies; comparison with non-drug treatments; and prospective economic analysis.

In conclusion, although the clinical benefits from docetaxel, gemcitabine, paclitaxel and vinorelbine appear relatively small, their benefit to patients with lung cancer appears to be worthwhile and cost effective. With important new evidence emerging, we recommend that our findings are periodically reviewed or revised.

\section{ACKNOWLEDGEMENT}

We thank the advisory group for advice and peer review of a draft of the original report for NICE, including: Dr J Baird, Director of Patient Care, Roy Castle Lung Cancer Foundation; Dr Frances Bowen, Consultant in Respiratory and General Medicine, Hammersmith Hospital, London; Dr Doug Coyle, Principal Investigator, Health Econom- ics, Clinical Epidemiology Unit, The Ottowa Hospital, Ottowa, Canada; Dr Michael Cullen, Consultant and Hon Reader in Medical Oncology, Cancer Centre at the Queen Elizabeth Hospital, Birmingham; Dr Anna Gregor, Consultant in Clinical Oncology, Western General Hospital, Edinburgh; Dr P Hopwood, Hon Consultant Psychiatrist, CRC Psychological Medicine Group, Christie Hospital, Manchester; Dr Fergus MacBeth, Chairman of COIN Lung Cancer Group/Member of Cochrane Lung Cancer Group, Consultant Oncologist, Velindre Hospital, Cardiff; Ms Kirsten Major, Health Economist, Ayr and Arran Health Board; Dr Ben Marshall, Consultant in Respiratory Medicine, Southampton General Hospital; Dr Martin Muers, Consultant in Respiratory Medicine, Leeds; Professor I E Smith, Professor of Cancer Medicine, The Royal Marsden Hospital, Sutton; Professor N Thatcher, Professor of Oncology, CRC Department of Medical Oncology, Christie CRC Research Centre, Christie Hospital, Manchester. We would also like to thank Dr Pam Royle and Ms Liz Hodson for their support with obtaining information; the South East Scotland Lung Study Group; SHPIC Costing Unit; and Dr Winter and colleagues at Kings Cross Hospital in Dundee.

\section{Authors' affiliations}

A Clegg, D A Scott, P Hewitson, M Sidhu, N Waugh, Southampton Health Technology Assessments Centre, Wessex Institute for Health Research and Development, University of Southampton, Southampton SOI6 7PX, UK

Conflicts of interest: none

Funding/support: This study was supported by the NHS R\&D HTA programme. The views and opinions expressed therein are those of the authors and do not necessarily reflect those of the NHS Executive.

\section{REFERENCES}

1 Coleman MP, Babb P, Domiecki P, et al. Cancer survival trends in England and Wales, 1971-1995: deprivation and NHS region. London: The Stationery Office, 1999.

2 Department of Health. NHS performance indicators. 2001 http://www.doh.gov.uk/nhsperformanceindicators.

3 Crook A, Duffy A, Girling DJ, et al. Survey on the treatment of non-small cell lung cancer (NSCLC) in England and Wales. Eur Respir J 1997; 10:1552-8.

4 Hardy JR, NobleT, Smith IE. Symptom relief with moderate dose chemotherapy (mitomycin-C, vinblastine and cisplatin) in advanced non small cell lung cancer. Br J Cancer 1989;60:764-6.

5 Marino $\mathbf{P}$, Pampallona $S$, Preatoni $A$, et al. Chemotherapy vs supportive care in advanced non-small-cell lung cancer: results of a meta-analysis of the literature. Chest 1994;106:861-5.

6 Non-small Cell Lung Cancer Cooperative Group. Chemotheraphy in non-small cell lung cancer: a meta-analysis using updated data on individual patient data from 52 randomised clinical trials. BM 1995;311:899-909.

7 Smith IE. Palliative chemotherapy for advanced non-small cell lung cancer. BM 1994;308:429-30

8 Souquet PJ, Chauvin F, Boissel JP, et al. Polychemotherapy in advanced non small cell lung cancer: a meta-analysis. Lancet 1993;342:19-21.

9 Thatcher N, Anderson H, Betticher DC, et al. Symptomatic benefit from gemcitabine and other chemotherapy in advanced non-small cell lung cancer: changes in performance status and tumour-related symptoms. Anticancer Drugs 1995;6:639-48

10 O'Brien MER, Cullen M. Managing patients with lung cancer. Guidelines must help bring us in line with European standards. BM 2000;320: 1604

11 Jaakimainen L, Goodwin PJ, Pater J, et al. Counting the costs of chemotherapy in a National Cancer Institute of Canada randomised tria in NSCLC. J Clin Oncol 1990:8:1301-9.

12 Scott D, Clegg A, Sidhu M, et al. Clinical and cost effectiveness of paclitaxel, docetaxel, gemcitabine and vinorelbine in lung cancer. Health Technology Assessment 2001 (in press).

13 Anderson H, Hopwood P, Stephens RJ, et al. Gemcitabine plus best supportive care (BSC) vs BSC in inoperable non-small cell lung cancer: a randomized trial with quality of life as the primary outcome. $\mathrm{Br} J$ Cancer 2000;83:447-53

14 Jadad AR, Moore A, Carroll D, et al. Assessing the quality of reports of randomised clinical trials: is blinding necessary? Control Clin Trials 1996;17:1-12.

15 Berthelot JM, Will BP, Evans WK, et al. Decision framework for chemotherapeutic interventions for metastatic non-small-cell lung cancer. J Natl Cancer Inst 2000;92:1321-9.

16 Smith TJ, Hillner BE, Neighbors DM, et al. Economic evaluation of a randomized clinical trial comparing vinorelbine, vinorelbine plus cisplatin, and vindesine plus cisplatin for non-small-cell lung cancer. J Clin Oncol 1995; 13:2166-73

17 Fossella FV, Devore R, Kerr RN, et al. Randomised phase III trial of docetaxel versus vinorelbine or ifosfamide in patients with advanced non-small-cell lung cancer previously treated with platinum-containing chemotherapy regimes. J Clin Oncol 2000;18:2354-62. 
18 Roszkowski K, Pluzanska A, Krzakowski M, et al. A multicenter, randomized, phase III study of docetaxel plus best supportive care versus best supportive care in chemotherapy-naive patients with metastatic or non-resectable localized non-small cell lung cancer (NSCLC). Lung Cancer 2000;27: 145-57.

19 Shepherd FA, Dancey J, Ramlau R, et al. Prospective randomized trial of docetaxel versus best supportive care in patients with non-small-cell lung cancer previously treated with platinum-based chemotherapy. J Clin Oncol 2000;18:2095-103.

20 Bokkel-Huinink WW, Bergman B, Chemaissani A, et al. Single-agent gemcitabine: an active and better tolerated alternative to standard cisplatin-based chemotherapy in locally advanced or metastatic non-small cell lung cancer. Lung Cancer 1999;26:85-94.

21 Cardenal F, Lopez-Cabrerizo MP, Anton A, et al. Randomized phase III study of gemcitabine-cisplatin versus etoposide-cisplatin in the treatment of locally advanced or metastatic non-small-cell lung cancer. J Clin Oncol 1999; 17:12-8

22 Crino L, Scagliotti GV, Ricci S, et al. Gemcitabine and cisplatin versus mitomycin, ifosfamide, and cisplatin in advanced non-small-cell lung cancer: a randomized phase III study of the Italian Lung Cancer Project. J Clin Oncol 1999;17:3522-30.

23 Perng RP, Chen YM, Ming L, et al. Gemcitabine versus the combination of cisplatin and etoposide in patients with inoperable non-small-cell lung cancer in a phase II randomized study. J Clin Oncol 1997;15:2097102.

24 Sandler AB, Nemunaitis J, Denham C, et al. Phase III trial of gemcitabine plus cisplatin versus cisplatin alone in patients with locally advanced or metastatic non-small-cell lung cancer. J Clin Oncol 2000;18:122-30

25 Cortes JE, Pazdur R. Docetaxel. J Clin Oncol 1995;13:2643-55.

26 Kelly K. New chemotherapy agents for small cell lung cancer. Chest 2000; 117:156-62S

27 Lorusso V, Carpagnano F, Frasci G, et al. Phase I/II study of gemcitabine plus vinorelbine as first-line chemotherapy of non-small-cell lung cancer. J Clin Oncol 2000;18:405-11.

28 Bonomi P, Kim K, Fairclough D, et al. Comparison of survival and quality of life in advanced non-small-cell lung cancer patients treated with two dose levels of paclitaxel combined with cisplatin versus etoposide with cisplatin: results of an Eastern Cooperative Oncology Group trial. J Clin Oncol 2000;18:623-31

29 Chang AY, Kim K, Glick J, et al. Phase II study of taxol, merbarone, and piroxantrone in stage IV non-small-cell lung cancer: The Eastern Cooperative Oncology Group. J Natl Cancer Inst 1993;85:388-94.

30 Gatzemeier U, von Pawel J, Gottfried M, et al. Phase III comparative study of high-dose cisplatin versus a combination of paclitaxel and cisplatin in patients with advanced non-small-cell lung cancer. J Clin Oncol 2000; 18:3390-9.

31 Giaccone G, Splinter TA, Debruyne C, et al. Randomized study of paclitaxel-cisplatin versus cisplatin-teniposide in patients with advanced non-small-cell lung cancer. The European Organization for Research and Treatment of Cancer Lung Cancer Cooperative Group. J Clin Oncol 1998; 16:2133-41

32 Postmus PE, Giaccone G, Debruyne C, et al. Results of the phase II EORTC study comparing paclitaxel/cisplatin with teniposide/cisplatin in patients with non-small cell lung cancer. EORTC Lung Cancer Cooperative Group. Semin Oncol 1996;23:10-3.

33 Ranson M, Davidson N, Nicolson M, et al. Randomized trial of paclitaxel plus supportive care versus supportive care for patients with advanced non-small cell lung cancer. J Natl Cancer Inst 2000;92:1074-80.

34 Baldini E, Tibaldi C, Ardizzoni A, et al. Cisplatin-vindesine-mitomycin (MVP) vs cisplatin-ifosfamide-vinorelbine (PIN) vs carboplatin-vinorelbine ( $\mathrm{CaN}$ ) in patients with advanced non-small-cell lung cancer (NSCLC): a FONICAP randomized phase II study. Italian Lung Cancer Task Force (FONICAP). Br J Cancer 1998:77:2367-70.

35 Colleoni $M$, Vicario $G$, Pancheri $F$, et al. A randomized phase II trial of cisplatinum plus mitomycin-C plus vinorelbine and carboplatin plus vinorelbine in advanced non-small cell lung cancer. Int $J$ Oncol 1997; 10:619-22.

36 Colucci G, Gebbia V, Galetta D, et al. Cisplatin and vinorelbine followed by ifosfamide plus epirubicin vs the opposite sequence in advanced unresectable stage III and metastatic stage IV non-small-cell lung cancer: a prospective randomized study of the Southern Italy Oncology Group (GOIM). Br J Cancer 1997;76:1509-17.
37 Comella P. Frasci G, De Cataldis G, et al. Cisplatin/carboplatin + etoposide + vinorelbine in advanced non-small-cell lung cancer: a multicentre randomised trial. Gruppo Oncologico Campano. Br J Cancer 1996;74:1805-11

38 Crawford J, O'Rourke M, Schiller JH, et al. Randomized trial of vinorelbine compared with fluorouracil plus leucovorin in patients with stage IV non-small-cell lung cancer. J Clin Oncol 1996;14:2774-84.

39 Depierre A, Chastang C, Quoix E, et al. Vinorelbine versus vinorelbine plus cisplatin in advanced non-small cell lung cancer: a randomized trial. Ann Oncol 1994;5:37-42

40 Furuse K, Fukuoka M, Kuba M, et al. Randomized study of vinorelbine (VRB) versus vindesine (VDS) in previously untreated stage IIIB or IV non-small-cell lung cancer (NSCLC). The Japan Vinorelbine Lung Cancer Cooperative Study Group. Ann Oncol 1996;7:815-20.

41 Le Chevalier T, Brisgand D, Douillard JY, et al. Randomized study of vinorelbine and cisplatin versus vindesine and cisplatin versus vinorelbine alone in advanced non-small-cell lung cancer: results of a European multicenter trial including 612 patients. J Clin Oncol 1994;12:360-7.

42 Lorusso V, Pezzella G, Catino AM, et al. Results of a clinical multicentric randomized phase II study of non-small cell lung cancer treated with vinorelbine-cisplatin versus vinorelbine alone. Int J Oncol 1995;6:65-8.

43 Martoni A, Guaraldi M, Piana E, et al. Multicenter randomized clinical trial on high-dose epirubicin plus cis-platinum versus vinorelbine plus cis-platinum in advanced non small cell lung cancer. Lung Cancer 1998:22:31-8.

44 Perol M, Guerin JC, Thomas P, et al. Multicenter randomized trial comparing cisplatin-mitomycin-vinorelbine versus cisplatin-mitomycin-vindesine in advanced non-small cell lung cancer. Groupe Francais de Pneumo-Cancerologie. Lung Cancer 1996;14:119-34.

45 The Elderly Lung Cancer Vinorelbine Italian Study Group (ELVIS) Effects of vinorelbine on quality of life and survival of elderly patients with advanced non-small-cell lung cancer. J Natl Cancer Inst 1999;91:66-72.

46 Wozniak AJ, Crowley JJ, Balcerzak SP, et al. Randomized trial comparing cisplatin with cisplatin plus vinorelbine in the treatment of advanced non-small-cell lung cancer: a Southwest Oncology Group study. J Clin Oncol 1998;16:2459-65.

47 Comella P, Frasci G, Panza N, et al. Cisplatin, gemcitabine, and vinorelbine combination therapy in advanced non-small-cell lung cancer: a phase II randomized study of the Southern Italy Cooperative Oncology Group. J Clin Oncol 1999;17:1526-34.

48 Comella P. Frasci $G$, Panza N, et al. Randomized trial comparing cisplatin, gemcitabine, and vinorelbine with either cisplatin and gemcitabine or cisplatin and vinorelbine in advanced non-small-cell lung cancer: interim analysis of a phase III trial of the Southern Italy Cooperative Oncology Group. J Clin Oncol 2000;18:1451-7.

49 Frasci G, Lorusso V, Panza N, et al. Gemcitabine plus vinorelbine versus vinorelbine alone in elderly patients with advanced non-small-cell lung cancer. J Clin Oncol 2000; 18:2529-36.

50 Kosmidis P, Mylonakis N, Dimopoulos A, et al. Combination chemotherapy with paclitaxel plus carboplatin versus paclitaxel plus gemcitabine in inoperable non-small cell lung cancer: a phase III randomized study. Preliminary results. Hellenic Cooperative Oncology Group. Semin Oncol 2000;27:3-8.

51 Perry MC, Ihde DC, Herndon JE, et al. Paclitaxel/ifosfamide or navelbine/ifosfamide chemotherapy for advanced non-small cell lung cancer: CALGB 9532. Lung Cancer 2000;28:63-8.

52 Silvestri G, Pritchard R, Welch HG. Preferences for chemotherapy in patients with advanced non-small cell lung cancer. BM patients with advance

53 NHS Centre for Reviews and Dissemination. Undertaking systematic reviews of research on effectiveness: CRD guidelines for those carrying out or commissioning reviews. CRD Report 4. 2nd ed. York. University of York. 2001

54 Juni P, Witschi A, Bloch R, et al. The hazards of scoring the quality of clinical trials for meta-analysis. JAMA 1999;282:1054-60.

55 Bero LA, Rennie D. Influences on the quality of published drug studies. Int J Technol Assess Health Care 2000;1 2:209-37.

56 Waugh N, Scott D. How should different life expectancies be valued? BM 1998:316:1316.

57 Manegold C, Bergman B, Chemaissani A, et al. Single-agent gemcitabine versus cisplatin-etoposide: early results of a randomised phase II study in locally advanced or metastatic non-small-cell lung cancer. Ann Oncol 1997;8:525-9.

58 Netten A, Curtis L. Unit costs of health and social care. PSSRU University of Kent, Canterbury: 2000. 